\title{
Choroidal Imaging with Swept Source Optical Coherence Tomography - A Review
}

\author{
Katarzyna Piasecka1 and Zofia Michalewska ${ }^{1,2}$ \\ 1. Third Municipal Hospital K Jonscher, Lodz, Poland; 2. Ophthalmic Clinic Jasne Blonia, Lodz, Poland
}

\begin{abstract}
The choroid provides up to $70 \%$ of blood and oxygen to the eye. Pathological changes of this vascular tissue may lead to malnutrition of the retina and therefore be involved in the pathogenesis of numerous retinal disorders. Swept source optical coherence tomography (SS-OCT) is a new development of a non-invasive imaging technique that uses a tunable laser source with a higher wavelength light than conventional spectral domain OCT (SD-OCT). This enables visualisation of tissues below the retinal pigment epithelium. Thanks to SS-OCT's ultrahigh speed and ultrahigh resolution it is possible to assess choroidal layers as well as to automatically create 3D maps of its thickness and volume. This review is to discuss how SS-OCT has improved our understanding of choroidal anatomy and function in various retinal and chorioretinal diseases. In future, detailed evaluation of choroid may play a crucial role in the diagnosis and management of various retinal diseases.
\end{abstract}

\section{Keywords}

Swept source OCT, SS-OCT, AMD, macular hole, epiretinal membrane, central serous chorioretinopathy, vitreomacular traction syndrome

Disclosure: Katarzyna Piasecka and Zofia Michalewska have no conflicts of interest to declare.

Received: 9 October 2014 Accepted: 7 November 2014 Citation: European Ophthalmic Review, 2014;9(2):132-6

Correspondence: Zofia Michalewska, Ophthalmic Clinic Jasne Blonia, Rojna 90, Lodz, Poland. E: zosia_n@yahoo.com

Optical coherence tomography (OCT) has revolutionised eye care and significantly improved our understanding of a number of retinal diseases. ${ }^{1}$ This non-invasive imaging technique utilises lowcoherence interferometry to create cross-sectional images, measuring backscattered or back-reflected light. The first commercially available devices used time domain modality (TD-OCT) and were designed to visualise the retina at $10 \mu \mathrm{m}$ resolution. They enabled us to obtain the first in vivo images of epiretinal membranes (ERMs), macular holes (MHS), macular oedema, drusen and other pathologies of the fovea..$^{2-6}$

Further rapid development resulted in the higher resolution and greater speed of spectral domain OCT (SD-OCT). ${ }^{7}$ The new scanning speed of approximately 50,000 A-scans/second allowed SD-OCT to capture highdensity 3D images of the structures of the eye. SD-OCT allowed us to reveal important clinical information in micro scale, as well as revealing the thickness of individual layers of the retina including the retinal nerve fibre layer (RNFL), retinal ganglion cell, plexiform, nuclear and photoreceptor layers by means of segmentation algorithms. The current commercially available SD-OCT devices use light wavelengths in the 800$870 \mathrm{~nm}$ range, which is appropriate for achieving highly detailed images of the retina at 5-6 $\mu \mathrm{m}$ resolution. However, the melanin contained in the retinal pigment epithelium (RPE) is highly scattered and absorbed in this range. ${ }^{8}$ This makes it difficult to visualise structures that lay beneath this layer, such as the choroid and choriocapillaris.

The choroid, which supplies up to $70 \%$ of blood to the eye, is essential for the retina to function normally. Choroidal abnormalities are strongly involved in the pathogenesis of a number of retinal diseases, such as choroidal neovascularisation, chorioretinal inflammatory diseases, central serous chorioretinopathy and others. Thus, an understanding of uveal anatomy is crucial for better diagnosis and the monitoring of treatment in these complicated eye conditions.

This review will discuss the most recent developments in OCT imaging using light wavelengths of 1,000-1,040 nm and how the new ability to penetrate below the RPE can improve our knowledge of choroidal morphology, in healthy and diseased eyes.

\section{The Choroid - What We Knew}

Histopathological studies revealed that choroidal layers include the inner and outer Bruch's membrane, the choriocapillaris, Sattler's layer, Haller's layer, delimited from the sclera by suprachoroidal layer (SCL) (lamina fusca, lamina suprachoroidea). Quantitative and qualitative in vivo assessment of the choroid has interested retina specialists, since its role in various chorioretinal diseases was established. Ex vivo histological studies on the thickness of chorioretinal layers revealed the thickest tissue underlies the macula region, thinning of the choroid as a result of ageing and thickening in the course of different chorioretinal pathologies.9.10 Due to their poor resolution and repeatability, traditional imaging techniques, such as ultrasonography or magnetic resonance imaging, provide limited insight into the diagnostic possibilities of choroidal abnormalities.1112 The analysis of choroidal vasculature by means of Indocyanine green (ICG) angiography allows us to examine blood circulation, from the arteries to the veins. ${ }^{13}$ Nevertheless, we were unable to visualise specific layers and it was not possible to take precise thickness or volume measurements.

\section{OCT in Choroidal Imaging}

The first attempts at choroidal imaging were reported using SD-OCT devices. Richard Spaide made the first attempt to visualise choroidal 
layers with commercially available SD-OCT systems by introducing enhanced depth imaging OCT (EDI-OCT). ${ }^{14}$ He discovered that an inverted image can be obtained by moving the device closer to the eye, positioning the choroid closer to the 'zero delay line', which enhances visualisation of tissues $500-800 \mu \mathrm{m}$ below the RPE.

To improve the signal-to-noise ratio, 50 to 100 B-scans of the same location have to be created and averaged together. As a result, choroidal tissue can be assessed at several submacular points..$^{15}$ This requires taking manual measurements with inbuilt digital calipers after an observer has identified the outer margin of the RPE and the choroidsclera junction. The method proved to have high reproducibility using several commercially available SD-OCT devices, showing good inter-visit and inter-observer correlation of results. ${ }^{16-18} 3 \mathrm{D}$ analysis was proposed by some authors to evaluate choroidal thickness and choroidal volume. ${ }^{19}$ However, it involved time-consuming manual choroid segmentation and the information obtained is limited to the subfoveal area. Tian et al. presented an automatic segmentation algorithm for spectralis SD-OCT; however, it is not commercially available to the clinicians. ${ }^{20}$ Furthermore, choroidal thickness and/or volume maps cannot be created automatically.

Swept source OCT (SS-OCT) is a novel device that uses photodetectors instead of spectrometers or line cameras and employs a tunable frequency swept laser with a narrowband light source. Near infrared light of 1,050 nm wavelength is less vulnerable to light scattering, thus creating the potential to penetrate more deeply into tissue compared with conventional SD-OCT devices, which operate at around the $800 \mathrm{~nm}$ range. ${ }^{21}$ As a result, B-scans can be acquired much faster (100,000 A-scans/second compared with 50,000 A-scans/second in conventional SD-OCT) and higher resolution is achieved ( $1 \mu \mathrm{m}$ versus 5-6 $\mu \mathrm{m}$ in SD-OCT). These improvements mean we can now obtain accurate 3D images of the retina and choroid and, for the first time, we are able to create choroidal thickness maps and automatically measure choroidal thickness. The device also provides a wider OCT scan of $12 \mu \mathrm{m}$ versus $9 \mu \mathrm{m}$ in conventional SD-OCT.

\section{Choroidal SS-OCT Imaging in Healthy Eyes}

The sharp, detailed images of what lies below the RPE enables us to distinguish the choroidal layers that are not visible when using conventional SD-OCT systems. ${ }^{22}$ The images correlate with histological findings: from the innermost Bruch's membrane, choriocapillaris, Sattler's layer (layer of medium-diameter blood vessels), Haller's layer (outermost layer of the choroid consisting of larger-diameter blood vessels) and the outer choroidoscleral boundary (CSB) (see Figure 1A).

The inner edge of the choroid, Bruch's membrane, is composed of the basal lamina of the RPE, collagen and elastic fibres. It compactly adjoins the choriocapillaris layer with no potential space or tissue between them.

The choroid's outermost border will be further called the CSB. We reported that the CSB is regular and follows the natural oval shape of the eye wall in all examined healthy volunteers and in myopic, disease-free eyes. ${ }^{23}$

In some cases, the SCL may be observed on the outer margin of the CSB. Some authors estimate this layer to be the innermost scleral layer, whilst others suggest it is the outermost choroidal layer. We observed that the SCL consists of two bands: the upper of which is hyperreflective; the lower of which is hyporeflective (see Figure 1B). When visualised by our group, this lower, hyporeflective band was judged to be the suprachoroidal space (SCS). ${ }^{23}$ Previously, this space

\section{Figure 1: Swept Source OCT in a Healthy Eye}

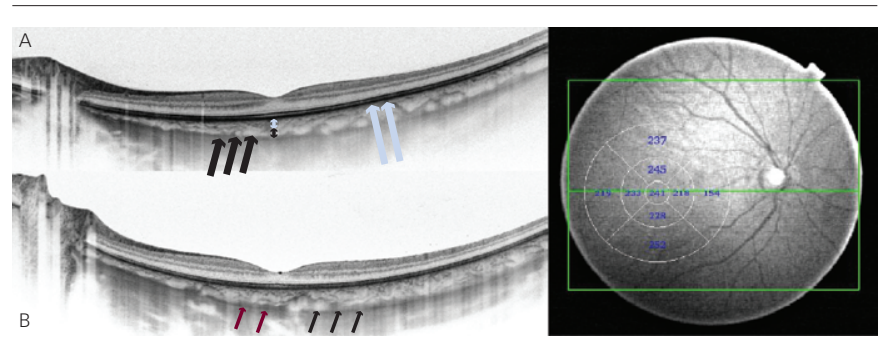

A. The internal structure of choroid from the inner to the outer margin is visible: choriocapillaris layer (white arrows), Sattler's layer consisting of medium-diameter blood vessels (white double arrowhead) and Haller's layer consisting of largediameter blood vessels (black double arrowhead). The outer choroidoscleral boundary is visible below the hyporeflective large choroidal vessels (black arrows) and follows the natural oval shape of the eye. B. Swept source optical coherence tomography (SS-OCT) of another healthy volunteer. Suprachoroidal layer is visible. It consists of two bands: the inner hyperreflective (black arrows); the outer hyporeflective (red arrows), estimated to be suprachoroidal space (SCS). C. SS-OCT healthy fundus view. Automated measurements of choroid thickness are performed and presented in nine segments according to the Early Treatment Diabetic Retinopathy Study.

had never been visualised in vivo in the macular area in a human eye without choroidal effusion. Short posterior ciliary arteries and vorticose veins penetrate through SCL.

Another significant improvement provided by commercially available SS-OCT is that automatic choroid thickness and volume measurements may be performed with excellent repeatability (see Figure 1C). ${ }^{24-26}$

As different investigators have reported various mean choroidal thicknesses, ranging from 191 to $354 \mu \mathrm{m}$, further studies are required. ${ }^{24,25,27-29}$ However, all the referenced authors agree that the choroid is thickest subfoveally and rapidly thins nasally to its thinnest point - about $1.5 \mathrm{~mm}$ from the fovea. ${ }^{24}$ This confirms earlier histological and EDI-OCT data.9.10,14-16

SS-OCT enables automatic choroidal volume measurement for the first time. Choroidal volume in the fovea is calculated as the volume of a $1,000 \mu \mathrm{m}$ diameter cylinder, the base of which is the central area of the Early Treatment Diabetic Retinopathy Study (ETDRS) Grid. Central choroidal volume in SS-OCT was reported at 0.159-0.17 $\mu \mathrm{m}$ and was lower than that reported with SD-OCT.24,25,27

In a normal population, choroidal thickness and volume has been negatively correlated with two independent variables: axial length and age. ${ }^{24,25}$ Correlation with refractive error is controversial, the literature reporting a 'no' to 'weak' positive correlation. ${ }^{25,27}$ Automated segmentation of choroidal measurements revealed for the first time that peripapillary and macular choroidal thickness and volume increase significantly after performing the water drinking test in healthy eyes. ${ }^{30}$

\section{SS-OCT in Retinal Diseases \\ Age-related Macular Degeneration}

In developed countries, age-related macular degeneration (AMD) is one of the leading causes of reduced visual acuity for those aged 60 years or over. ICG angiography studies showed hypoperfusion in choroidal circulation in eyes with wet AMD. ${ }^{31}$ Although this suggests that choroidal function is altered in the course of this disease, the exact contribution of blood circulation in AMD pathogenesis is still being investigated.

Using Cirrus ${ }^{\mathrm{TM}}$ HD-OCT, Manjunath et al. confirmed choroidal thinning in eyes with dry AMD and further reduction in eyes with wet AMD compared with age-matched patients. ${ }^{32} \mathrm{He}$ found no significant correlation between 


\section{Figure 2: Swept Source OCT in Age-related Macular Degeneration}

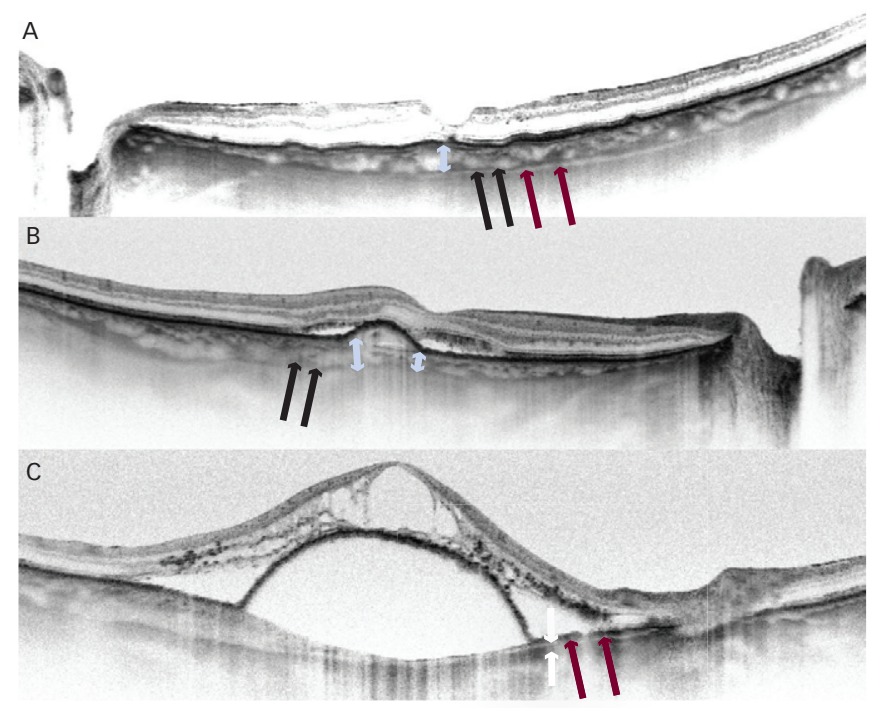

Choroidal thickness (white doublehead arrow) in dry age-related macular degeneration $(A M D)(A)$ and in neovascular $A M D(B, C)$. Suprachoroidal layer is visible (A: red arrows; $C$ white and red arrows). It consists of two bands, the inner hyperreflective (black arrows), and the outer hyporeflective (red arrows), estimated to be suprachoroidal space.

choroidal thickness and the number of anti-VEGF injections, disease duration, nor visual acuity.

SS-OCT analysis showed no thinning in the early stage of AMD (defined as the presence of soft drusen greater than $125 \mu \mathrm{m}$ in diameter, pigment changes or drusenoid pigment epithelial detachment). ${ }^{33}$ Other authors also demonstrated a relationship between AMD severity and choroidal thickness reduction. ${ }^{34}$ Choroidal thinning was observed in patients treated with anti-VEGF agents. ${ }^{35,36}$

Our observations are coincident with presented observations. Although we have reported an increase in the thickness of Haller's layer in early wet AMD, an overall decrease in choroidal thickness is observed as the disease progresses (see Figure 2).22,37 Sattler's layer becomes almost invisible, and Haller's layer and the choriocapillaries are atrophic. The clinical implications of these findings need to be elucidated in the future, but it is suggested that insufficient blood supply may contribute to RPE atrophy and degeneration.

Changes in choroidal thickness are not the only phenomenon noticed in AMD progression. Morphological analysis reveals the existence of an irregular CSB line in $25 \%$ of eyes with dry AMD and in $50 \%$ of eyes with wet $\mathrm{AMD}^{23}$ Additionally, we noted CSB irregularities in all cases of wet $A M D$, which were qualified for reinjection of anti-vascular endothelial growth factor (VEGF) (unpublished data).

We observed the SCL and SCS in $20 \%$ of eyes with wet AMD and in $50 \%$ of eyes with dry AMD. ${ }^{23}$

\section{Age-related Choroidal Atrophy}

Age-related choroidal atrophy (ARCA) of tissue was first described by Spaide et al., who demonstrated that choroidal thinning was associated with choroidal small vessel loss in some non-myopic elderly patients. ${ }^{38}$ In ARCA, unlike in geographic atrophy and AMD, the RPE layer remains normal. Choroidal thickness observed with EDI-OCT was reported to be markedly decreased. ${ }^{38}$ AMD and ARCA affect older adults and may coexist. ${ }^{39}$ This suggests that ARCA may contribute in dry AMD pathogenesis. Future SSOCT studies may enhance our understanding of ARCA and its implications.

\section{Central Serous Chorioretinopathy}

Central serous chorioretinopathy (CSCR) is characterised by serous detachment of neurosensory retina from the RPE. When Gass described CSCR in 1967, he proposed that it was the choriocapillaris hyperpermeability that was the source of the leak. ${ }^{40}$ Later, studies with ICG angiography and fluorescein angiography showed a generalised disruption of choroidal vasculature in eyes affected with CSCR, with diffuse hyperpermeability, confirming Gass' original idea. ${ }^{41}$ EDI-OCT demonstrated very thick choroid in patients with CSCR (505 $\pm 124 \mu \mathrm{m})$. Further SS-OCT studies revealed that mean choroidal thickness was increased in classic, chronic and multifocal CSCR (see Figure 3), and was greater in areas with choroidal vascular hyperpermeability and in areas where punctate hyperfluorescent spots showed up on ICG angiography than in unaffected areas. ${ }^{42,43}$ Detailed SS-OCT analysis of 15 eyes with CSCR demonstrated focally enlarged vessels at the level of choriocapillaris in $53 \%$ of cases, as well as focal or diffuse dilation in Sattler's and Haller's layers in all analysed eyes. ${ }^{44}$

Choroidal thickening reportedly reduces following photodynamic therapy but not after laser photocoagulation. ${ }^{45}$ This suggests that laser treatment does not influence the underlying problem, which involves the choroid.

Interestingly, increased choroidal thickening was also reported in eyes after spontaneous CSCR resolution. ${ }^{46}$ We assume that hyperpermeability leads to both: choroid thickening and to CSCR recurrences. After the spontaneous resolution we can see thick choroid but there is no test to check vascular permeability. In our opinion this is an indirect proof of persistent vascular abnormalities in choroid. CSCR recurrences are frequent after spontaneous resolution of CSCR.

\section{Vitreoretinal Interface Diseases}

We recently analysed the morphology of outer choroidal margins in patients with different vitreoretinal disorders, including ERM, vitreomacular traction (VMT) syndrome and MHs. ${ }^{23,47-49}$ Unlike in healthy eyes, an irregular outer CSB line, not following the natural shape of the eye wall, may be noted in different vitreoretinal interface diseases (see Figure 4). This is much more frequently observed in eyes qualified for surgery. For example, irregular CSB was observed in $25 \%$ of eyes with ERM, but in $90 \%$ of eyes with ERM qualified for surgery. These irregularities may reflect the activity of the process. Irregular CSB is also observed in $40-59 \%$ of eyes with full-thickness $\mathrm{MH}$ and $60 \%$ of eyes with lamellar MH. Additionally, the SCL and SCS were noted in $34 \%$ of eyes with full-thickness $\mathrm{MH}, 44 \%$ of $\mathrm{MH}$ fellow eyes, in $60 \%$ of eyes with VMT syndrome and $19 \%$ of eyes with ERMs. Progression in VMT syndrome may be more rapid than in full-thickness MHs. ERMs are a 'static' disease: it was reported that patients lose one visual acuity line every 5 years. ${ }^{50}$ Further studies are required to investigate what role the degree of visibility of the SCL and SCS might play in ophthalmology.

An interesting finding in patients with ERM is that choroidal thickening is observed in the foveal area compared with normal age and refractiveerror-matched subjects. We cannot exclude that increased thickness is associated with macular thickening, which results in higher oxygen and blood demand. Another hypothesis may be that choroidal thickening is primary to macular changes and causes retinal thickening. ${ }^{47}$ Choroidal thickness decreases after vitrectomy with ILM peeling in eyes with idiopathic ERM. ${ }^{48}$ 
The role of the choroid in MHs is elusive. Some investigators reported reduced choroidal thickness in EDI-OCT studies of patients with fullthickness $\mathrm{MH}$. ${ }^{51,52}$ This leads to the hypothesis that reduced vasculature, insufficient blood and oxygen supply, may increase the susceptibility of the fovea to traction. ${ }^{53}$ Subsequent EDI-OCT reports did not confirm choroidal thinning in patients with idiopathic $\mathrm{MH}^{.}{ }^{54}$

\section{SS-OCT in Glaucoma}

Glaucomatous neuropathy is one of the leading causes of blindness in the world. Histopathological findings in animals, cadavers and enucleated eyes suggest that axonal damage and subsequent retinal ganglion cells apoptosis are associated with lamina cribrosa (LC) thinning and deformation. ${ }^{55-57}$ It was demonstrated with EDI-OCT that LC in glaucoma patients is thinner than in normal subjects. ${ }^{58}$ However, the posterior laminar surface is not visualised clearly using this technique. 3D raster scanning with ideal sampling density in SS-OCT enables detailed analysis of the optic disc including LC. Focal LC defects, which corresponded with neuroretinal rim thinning and with visual field defects, were observed in glaucoma patients. ${ }^{59}$

Glaucoma affects choroidal thickness as well. Choroidal thinning was observed in overall, peripapillar and macular region in glaucoma patients compared with healthy controls..$^{60}$

\section{Choroidal Imaging for Non-ophthalmological Conditions}

The choroid has the highest blood flow per unit weight of any tissue in the body. Being composed mainly of blood vessels, the choroid should be very vulnerable to vascular changes, such as prolonged vasospasm.

Tobacco smoke exposure has a vasoconstrictive effect on cerebral vasculature. Some authors demonstrated a decrease in the flow velocity of the ophthalmic artery, central retinal artery and posterior ciliary artery, suggesting a significant increase in vascular resistance. .11,62 $^{2}$ Therefore, the thinning of the choroid layer is an expected clinical outcome in chronic tobacco smoking. SD-OCT studies showed that the mean choroidal thickness at the fovea decreased significantly at 1 hour and at 3 hours following smoking. ${ }^{63}$ Sigler et al., in a multivariate analysis of variance, reported that a history of smoking was significantly associated with a thinner choroid in patients at all ages (mean CT: 148 $\pm 63 \mu \mathrm{m}$ versus $181 \pm 65 \mu \mathrm{m}$ for non-smokers). ${ }^{64}$ Choroidal thinning was greater in smoking- than non-smoking early-AMD subjects. High positive correlation was reported between the pack-years of smoking and a thinner choroid $(p<0.001)$.

Similar findings are suggested in migraine patients. Migraine is known to be a neurovascular disease, in which vasospasm occurs in the tissues outside the brain, especially in the retina layer, concurrent with a reduction in brain blood flow. ${ }^{65,66}$ Blood flow at the level of the central retinal artery and posterior ciliary artery is decreased, as was shown in colour Doppler ultrasonography. ${ }^{67}$ Recent SD-OCT studies confirm choroidal thinning in patients with migraine, which is greater in patients with aura than those without aura. ${ }^{68}$ Choroidal changes were followed by decrease in the RNFL thickness in all patients. Moreover, thinning of the ganglion cell layer was reported in the group with aura.

The roles of hypoperfusion and ischaemic lesions have also been discussed in some neurodegenerative diseases. RNFL thinning

\section{Figure 3: Swept Source OCT in Central Serous Chorioretinopathy (CSCR) Reveals Thickening of the Choroid (White Doublehead Arrow)}

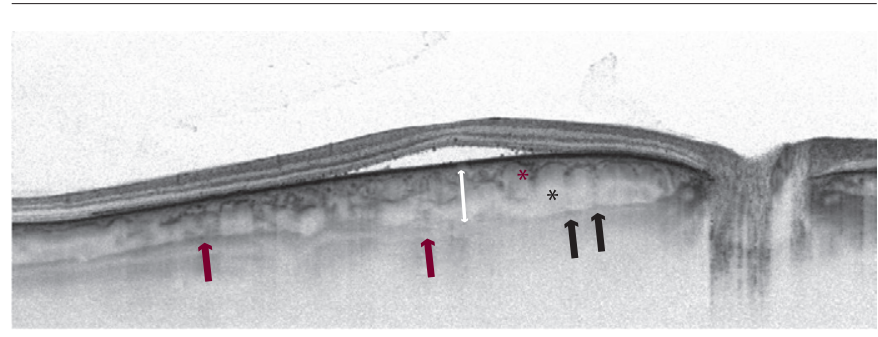

Diffuse vascular dilation is visible in all choroidal layers - enlarged vessels of Sattler's layer (red star) merge with dilated vessels of Haller's layer (black star). Suprachoroidal layer is visible (red arrows). It consists of two bands, the inner hyperreflective (black arrows), and the outer hyporeflective (red arrows), estimated to be suprachoroidal space (SCS).

\section{Figure 4: Swept Source OCT Imaging in Different Vitreoretinal Disorders}
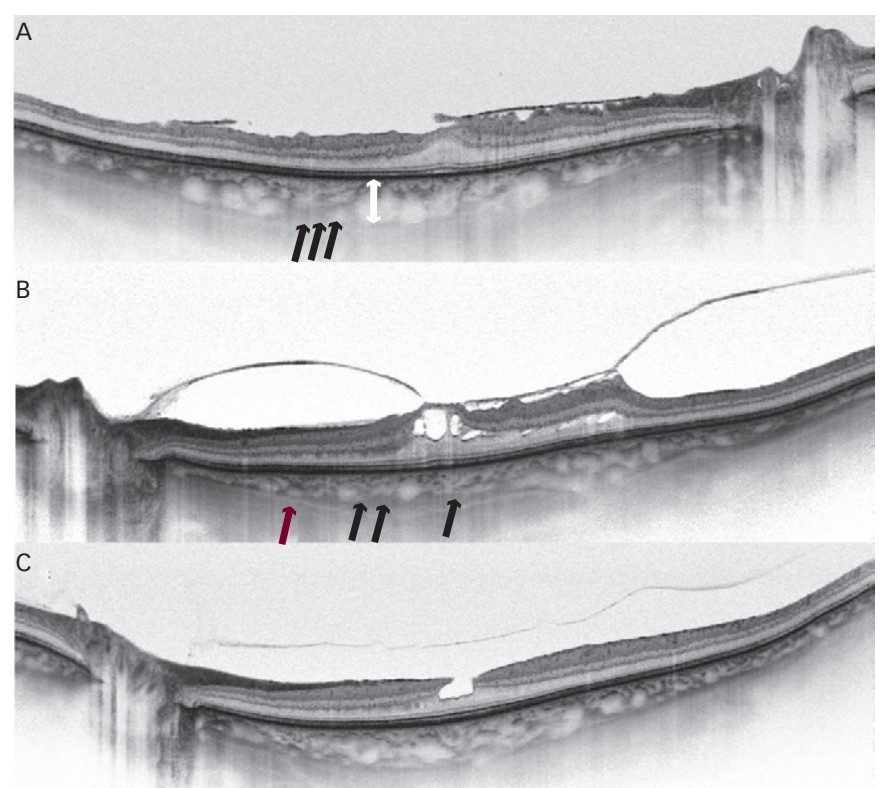

A: Epiretinal membrane. Choroidal thickness is marked with a white doublehead arrow. The outer choroidoscleral boundary is regular and suprachoroidal layer is visible (black arrows) B: Vitreomacular traction syndrome. Irregular outer choroidoscleral boundary (black arrows) with visible suprachoroidal space (red arrow). C: Lamellar macular hole with detached posterior vitreous.

was reported in Alzheimer's disease, and Parkinson's disease and multiple sclerosis. ${ }^{69-71}$ Retinal changes may be secondary to insufficient blood supply.

No analysis in SS modality has been performed on choroidal structure and thickness in mentioned situations associated with reduced choroidal blood flow. We believe that SS-OCT may contribute additional information how choroid changes with prolonged hypoperfusion.

The new possibility to examine tissues below the RPE in greater detail are of tremendous value and will likely become more widely utilised in neuro-ophthalmology and other fields where they can provide deeper insight into the pathogenesis, treatment and follow up of neurodegenerative diseases. OCT angiography with the use of higher wavelight in SS demonstrated recently reduced blood flow index in optic nerve head in patients with multiple sclerosis and further flow index reduction after optic neuritis. ${ }^{72}$ SS-OCT might provide more details on choroidal pathophysiology in the intercourse of neurodegenerative diseases. 


\section{Conclusion}

Choroidal imaging marks a milestone in posterior segment OCT visualisation. It not only provides quantitative evaluation of choroidal thickness and volume, but also makes qualitative assessment possible. For the first time, we can distinguish separate choroidal layers and discover new morphological characteristics of particular diseases. Now that this ultrahigh speed and ultrahigh resolution technique is commercially available, our diagnostic and monitoring abilities in chorioretinal diseases are better than ever. In the future, clinical application of SS-OCT may involve various neurological conditions as well as vitreoretinal pathologies.
1. Huang D, Swanson EA, Lin CP, et al., Optical coherence tomography, science, 1991:254:1178-1.

2 Puliafito CA, Hee MR, Lin CP, et al., Imaging of macula diseases with optical coherence tomography, Ophthalmology, 1995;102:217-29.

3. Hee MR, Puliafito CA, Wong C, et al., Optical coherence tomography of macular holes, Ophthalmology, 1995;102:748-56

4. Hee MR, Puliafito CA, Wong C, et al., Quantitative assessment of macular edema with optical coherence tomography, Arch Ophthalmol, 1995;113:1019-29.

5. Hee MR, Puliafito CA, Wong C, et al., Optical coherence tomography of central serous chorioretinopathy, Am J Ophthalmol, 1995:120:65-74.

6. Hee MR, Baumal CR, Puliafito CA, et al., Optical coherence tomography of age-related macular degeneration and choroidal neovascularization, Ophthalmology, and choroidal neovas

7. Wojtkowski M, Srinivasan V, Fujimoto JG, et al., Threedimensional retinal imaging with high-speed ultrahig resolution optical coherence tomography, Ophthalmology, 2005;112:1734-46.

8. Wolbarsht ML, Walsh AW, George G, Melanin, a unique biological absorber, Appl Opt, 1981;20:2184-6.

9. Ramrattan RS, van der Schaft TL, Mooy CM, et al., Morphometric analysis of Bruch's membrane, the choriocapillaris, and the choroid in aging, Invest Ophthalmol Vis SCi, 1994;35:2857-64.

10. Curcio CA, Messinger JD, Sloan KR, et al., Human chorioretinal layer thicknesses measured in macula-wide, high-resolution histologic sections, Invest Ophthalmol Vis Sci, high-resolution histo

11. Coleman DJ, Lizzi FL, In vivo choroidal thickness measurement, Am J Ophthalmol, 1979:88:369-75.

12. Townsend KA, Wollstein G, Schuman JS, Clinical application of MRI in ophthalmology, NMR Biomed, 2008;21:997-1002.

13. Guyer DR, Puliafito CA, Mones JM, Digital indocyanine-green angiography in chorioretinal disorders, Ophthalmology, 1992;99:287-91.

14. Spaide RF, Koizumi H, Pozzoni MC, Enhanced depth imaging spectral-domain optical coherence tomography, Am J Ophthalmol, 2008;146:496-500

15. Manjunath V, Taha M, Fujimoto JG, Duker JS, Choroida thickness in normal eyes measured using Cirrus HD optical coherence tomography, Am I Ophthalmol, 2010;150:325-9.

16. Yamashita T, Yamashita T, Shirasawa M, et al., Repeatability and reproducibility of subfoveal choroidal thickness in normal eyes of Japanese using different SD-OCT devices, Invest Ophthalmol Vis Sci, 2012;53:1102-7.

17. Branchini L, Regatieri CV, Flores-Moreno I, et al, Reproducibility of choroidal thickness measurements across three spectral domain OCT systems, Ophthalmology , 2012;119:119-23.

18. Ikuno Y, Maruko I, Yasuno $Y$, et al., Reproducibility of retinal and choroidal thickness measurements in enhanced depth imaging and high-penetration optical coherence tomography, Invest Ophthalmol Vis Sci, 2011;52:5536-40.

19. Barteselli G, Chhablani J, El-Emam S, et al., Choroidal volume variations with age, axial length, and sex in healthy subjects: a three-dimensional analysis, Ophthalmology, 2012:119:2572-8.

20. Tian 」. Marziliano P, Baskaran M, et al., Automatic segmentation of the choroid in enhanced depth imaging optical coherence tomography images, Biomedical Optics Express, 2013;4:397-411

21. Unterhuber B, Povazay B, Hermann $\mathrm{H}$, In vivo retinal optical coherence tomography at $1040 \mathrm{~nm}$-enhanced penetration into the choroid, Opt Express, 2005; 13:3252-8.

22. Michalewska Z, Michalewski J, Nawrocki J, Going deeper and going wider, Retinal Physician, 2013;3:42-8.

23. Michalewska Z, Michalewski J, Nawrocka Z, et al., Lamina suprachoroidea and suprachoroidal space delineating the outer margin of the choroid in swept-source OCT, BioMed Research International volume 2014 (2014). Article ID 639160, 7 pages

24. Michalewski J, Michalewska Z, Nawrocka Z, et al., Correlation of choroidal thickness and volume measurements with axial length and age using swept source optical coherence tomography and optical low-coherence reflectometry, Biomed Res Int, 2014:2014:639160.

25. Hirata M, Tsujikawa A, Matsumoto A, et al., Macular choroidal thickness and volume in normal subjects measured by swept-source optical coherence tomography, Invest ophthalmol Vis Sci, 2011;2:4971-8.

26. Mansouri K, Medeiros FA, Tatham AJ, et al., Evaluation of retinal and choroidal thickness by swept-source optical coherence tomography: repeatability and assessment of artifacts, Am J Ophthalmol, 2014;157:1022-32.

27. Ikuno Y, Kawaguchi K, Nouchi T, Yasuno Y, Choroidal thickness in healthy Japanese subjects, Invest Ophthalmol Vis SCi, 2010;51:2173-6.

28. Ruiz-Medrano J, Flores-Moreno I, Peña-García P, et al., Macular choroidal thickness profile in a healthy population measured by swept-source optical coherence tomography, Invest Ophthalmol Vis Sci, 2014:55:3532-42.

29. Agawa T, Miura M, Ikuno Y, et al., Choroidal thickness measurement in healthy Japanese subjects by threedimensional high-penetration optical coherence tomography, Graefes Arch Clin Exp Ophthalmol, 2011;249:1485-92.

30. Mansouri K, Medeiros FA, Marchase N, et al., Assessment of choroidal thickness and volume during the water drinkin test by swept-source optical coherence tomography, Ophthalmology, 2013;120:2508-16.

31. Koizumi H, lida T, Saito M, et al., Choroidal circulatory disturbances associated with retinal angiomatous proliferation on Indocyanine green angiography, Graefes Arch Clin Exp Ophthalmol, 2008;246:515-20.

32. Manjunath V, Goren J, Fujimoto JG, Duker JS, Analysis of choroidal thickness in age-related macular degeneration using spectral-domain optical coherence tomography, Am J Ophthalmol, 2011;152:663-8.

33. Wood A, Binns A, Margrain T, et al., Retinal and choroidal thickness in early age-related macular degeneration, Am J Ophthalmol, 2011:152:1030-8.

34. Switzer DW Jr, Mendonca LS, Saito M, et al., Segregation of ophthalmoscopic characteristics according to choroida thickness in patients with early age-related macula degeneration, Retina, 2012;32:1265-71.

35. Yamazaki T, Koizumi H, Yamagishi T, Kinoshita SO, Subfoveal choroidal thickness after ranibizumab therapy for neovascular age-related macular degeneration: 12-month results, Ophthalmology, 2012;119:1621-7.

36. Rahman W, Chen FK, Yeoh J, da Cruz L, Enhanced depth imaging of the choroid in patients with neovascular Agerelated macular degeneration treated with anti-VEGF therapy versus untreated patients, Graefes Arch Clin Exp Ophthalmo 2013;251:1483-8

37. Michalewska Z Swept-source OCT: Taking imaging deeper and wider, Retina Today, 2014;11/12:S:4.

38. Spaide RF, Age-related choroidal atrophy, Am J Ophthalmol, 2009;147:801-10.

39. Switzer DW Jr, Mendonca LS, Saito M, et al., Segregation of ophthalmoscopic characteristics according to choroidal thickness in patients with early age-related macular degeneration, Retina, 2012;32:1265-71.

40. Gass ID, Pathogenesis of disciform detachment of the neuroepithelium, Am J Ophthalmol, 1967;63(Suppl.):1-139.

1. Gemenetzi M, De Salvo G, Lotery AJ, Central serous chorioretinopathy: an update on pathogenesis and treatment, Eye (Lond), 2010;24:1743-56.

42. Jirarattanasopa P, Ooto S, Tsujikawa A, et al., Assessment of macular choroidal thickness by optical coherence tomography and angiographic changes in central serous chorioretinopathy, Ophthalmology, 2012;119:1666-78.

43. Razavi S, Souied EH, Cavallero E, et al., Assessment of choroidal topographic changes by swept source optical coherence tomography after photodynamic therapy for central serous chorioretinopathy, Am J Ophthalmol, 2014; 157:852-60

44. Ferrara D, Mohler KJ, Waheed N, et al., En face enhanceddepth swept-source optical coherence tomography features of chronic central serous chorioretinopathy, Ophthalmology, 2014;121:719-26.

45. Maruko I, lida T, Sugano Y, et al., Subfoveal choroidal thickness after treatment of central serous chorioretinopathy Ophthalmology, 2010;117:1792-9.

46. Kang NH, Kim YT, Change in subfoveal choroidal thickness in central serous chorioretinopathy following spontaneous resolution and low-fluence photodynamic therapy, Eye (Lond), 2013;27:387-91.

47. Michalewska Z Michalewski 」, Nawrocki J, Swept Source OCT: Wide-field simultaneous choroid, retina and vitreous visualization, Retina Today, 2013;9:50-6.
48. Michalewska Z, Michalewski 」, Zawislak E, et al , Choroidal thickness measured with swept source OCT before and after vitrectomy with ILM peeling for idiopathic epiretinal membranes, $R$.

49. Michalewska Z, Michalewski J, Sikorski BL, et al., A study of macular hole formation by serial spectral optical coherence tomography. Clin Exp Ophthalmol. 2008;37:373-83.

50. Theodossiadis PG, Grigoropoulos VG, Emfietzoglou I, et al., Evolution of lamellar macular hole studied by optical coherence tomography, Graefes Arch Clin Exp Ophthalmol, 2009;247:13-20.

51. Reibaldi M, Boscia F, Avitabile T, et al., Enhanced depth imaging optical coherence tomography of the choroid in idiopathic macular hole: A cross-sectional prospective study, Am J Ophthalmol, 2011:151:112-7.

52. Zeng J, Li J, Liu R, et al., Choroidal thickness in both eyes of patients with unilateral idiopathic macular hole, Ophthalmology, 2012;119:2328-33.

53. Aras C, Ocakoglu O, Akova N, Foveolar choroidal blood flow in idiopathic macular hole, Int Ophthalmol, 2004;25:225-31.

54. Schaal KB, Pollithy S, Dithmar S, Is choroidal thickness of importance in idiopathic macular hole?, Ophthalmologe 2012;109:364-8

55. Quigley HA, Addicks EM, Green WR, Maumenee AE, Optic nerve damage in human glaucoma. II. The site of injury and susceptibility to damage, Arch Ophthalmol, 1981;99:635-49.

56. Quigley HA, Hohman RM, Addicks EM, et al., Morphologic changes in the lamina cribrosa correlated with neural loss in open-angle glaucoma, Am J Ophthalmol, 1983;95:673-91.

57. Bellezza AJ, Rintalan CJ, Thompson HW, et al., Deformation of the lamina cribrosa and anterior scleral canal wall in early experimental glaucoma, Invest Ophthalmol Vis Sci. 2003;44:623-37.

58. Park HY, Jeon SH, Park CK, Enhanced depth imaging detects lamina cribrosa thickness differences in normal tension glaucoma and primary open-angle glaucoma, Ophthalmology, 2012;119:10-20

59. Takayama K, Hangai M, Kimura Y, et al., Three-dimensional imaging of lamina cribrosa defects in glaucoma using sweptsource optical coherence tomography, Invest Ophthalmol Vis Sci, 2013;54:4798-807.

60. Zhang C, Tatham AJ, Medeiros FA, et al., Assessment of choroidal thickness in healthy and glaucomatous eyes using swept source optical coherence tomography, PLoS One, 2014;9:e109683

61. Steigerwalt RD Jr, Laurora G, Incandela L, et al., Ocular and orbital blood flow in cigarette smokers, Retina, 2000:20:394-7.

62. Tamaki Y, Araie M, Nagahara M, et al., The acute effects of cigarette smoking on human optic nerve head and posterio fundus circulation in light smokers, Eye (Lond), 2000;14:67-72.

63. Sizmaz S, Küçükerdönmez C, Pinarci EY, et al., The effect of smoking on choroidal thickness measured by optical coherence tomography, Br J Ophthalmol, 2013;97:601-4.

64. Sigler EJ, Randolph JC, Calzada JI, Charles S, Smoking and choroidal thickness in patients over 65 with early-atrophic age-related macular degeneration and normals, Eye (Lond) 2014;28:838-46.

65. Killer HE, Forrer A, Flammer J, Retinal vasospasm during an attack of migraine, Retina, 2003:23:253-4.

66. Beversdof D, Stommel E, Allen C, et al., Recurrent branch retinal infarcts in association with migraine, Headache 1997:37:396-9.

67. Kara SA, Erdemoglu AK, Karadeniz MY, Altinok D, Color Doppler sonography of orbital and vertebral arteries in migraineurs without aura, J Clin Ultrasound, 2003;31:308-14.

6. Ekinci M, Ceylan E, Cagatay HH, et al., Retinal nerve fibre layer, ganglion cell layer and choroid thinning in migraine with aura, BMC Ophthalmol, 2014;14:75

69. Kirbas S, Turkyilmaz K, Anlar O, et al., Retinal nerve fiber layer thickness in Alzheimer disease, I Neuroophthalmol, 2013;33:58-61.

70. Kirbas S, Turkyilmaz K, Tufekci A, Durmus M, Retinal nerve fiber layer thickness in Parkinson disease, I Neuroophthalmol, 2013:33:62-5

71. Galetta KM, Calabresi PA, Frohman EM, Balcer LJ, Optical coherence tomography (OCT): imaging the visual pathway as a model for neurodegeneration, Neurotherapeutics, 2011:8:117-32.

72. Wang $X$, Jia $Y$, Spain $R$, Potsaid $B$, et al., Optical coherence tomography angiography of optic nerve head and parafove in multiple sclerosis, Br J Ophthalmol, 2014;98:1368-73. 